\title{
Spectral Response of UV Photodetectors for Barium Fluoride Crystal Readout
}

\author{
Liyuan Zhang, Member, IEEE, Chen Hu, Member, IEEE, James Oyang, Bertrand Echenard, David Hitlin, \\ Xuebin Qiao, Jason Trevor, and Ren-Yuan Zhu, Senior Member, IEEE
}

\begin{abstract}
Because of its fast scintillation peaked at $220 \mathrm{~nm}$ with decay time of less than $0.6 \mathrm{~ns}$ barium fluoride crystals have attracted a broad interest in the community pursuing ultrafast detectors for future HEP experiments and $\mathrm{GHz}$ hard X-ray imaging for future XFEL facilities. A crucial issue for its application is the spectral response of photodetectors down to VUV. In this paper, we compare quantum efficiency and photon detection efficiency spectra measured for several UV photodetectors down to $200 \mathrm{~nm}$. Their figures of merit on the detection efficiency for the fast component and the suppression of the slow component are also discussed.
\end{abstract}

\section{INTRODUCTION}

$\mathrm{B}$ ECAUSE of its fast cross-luminescence [1-7] peaked at 220 $\mathrm{nm}$ with decay time of less than 0.6 ns [8-15] barium fluoride $\left(\mathrm{BaF}_{2}\right)$ crystals have attracted a broad interest in the community pursuing ultrafast calorimetry for future high energy and nuclear physics experiments [14,19] and for $\mathrm{GHz}$ hard X-ray imaging for future X-ray Free Electron Laser (XFEL) facilities [9,13]. One crucial issue preventing $\mathrm{BaF}_{2}$ application is its slow scintillation component peaked at $300 \mathrm{~nm}$ due to self-trapped excitons with decay time of $600 \mathrm{~ns}$ and an intensity of more than five times of the fast component, which causes pileup and readout noise in a high-rate environment.

A $\mathrm{BaF}_{2}$ crystal calorimeter was baselined for the Mu2e experiment [14] and was replaced by an undoped CsI crystal calorimeter mainly due to this slow component [15]. It is also well known that undoped CsI survives the Mu2e radiation environment where an ionization dose up to a few tens krad but not a few hundred krad expected by Mu2e-II. $\mathrm{BaF}_{2}$, on the other hand, is found more radiation hard against an ionization dose up to 200 Mrad [16], a proton fluence up to $1 \times 10^{15} \mathrm{p} / \mathrm{cm}^{2}$ [17] and a neutron fluence up to $9 \times 10^{15} \mathrm{n}_{\mathrm{eq}} / \mathrm{cm}^{2}$ [18]. To cope with the unprecedented event rate, a $\mathrm{BaF}_{2}$ crystal-based ultrafast calorimeter is proposed for the Mu2e-II experiment [19]. One crucial issue is the pile-up and readout noise caused by the slow component. R\&D has been going-on along two directions to address this issue. One is selective rare earth or transition metal doping in $\mathrm{BaF}_{2}$ crystals [20-32]. The other is to use solar-blind photodetectors sensitive only to the fast component at $220 \mathrm{~nm}$ but not the slow component at $300 \mathrm{~nm}$ [27, 33-35].

Manuscript received Dec 12, 2021. This work was supported by the U.S. Department of Energy, Office of High Energy Physics program under Award No. DE-SC0011925.
Progresses have been achieved along both directions. Fig. 1 shows X-ray excited emission spectra measured for $\mathrm{BaF}_{2}$ crystal samples with different yttrium doping level, showing consistent fast scintillation component, and significantly suppressed slow component with increased yttrium doping level [31]. Recent investigation also shows that solar-blind UV photodetectors are needed to further suppress the slow component and reduce radiation induced readout noise to a level of less than $1 \mathrm{MeV}$ for $\mathrm{BaF}_{2}$ crystals of $\mathrm{Mu} 2 \mathrm{e}$ size [36]. In this paper, we report quantum efficiency (QE) and photon detection efficiency (PDE) spectra measured for several UV/VUV photodetectors down to $200 \mathrm{~nm}$. Their figure of merit on the detection efficiency for the fast component and the slow component suppression is also discussed.

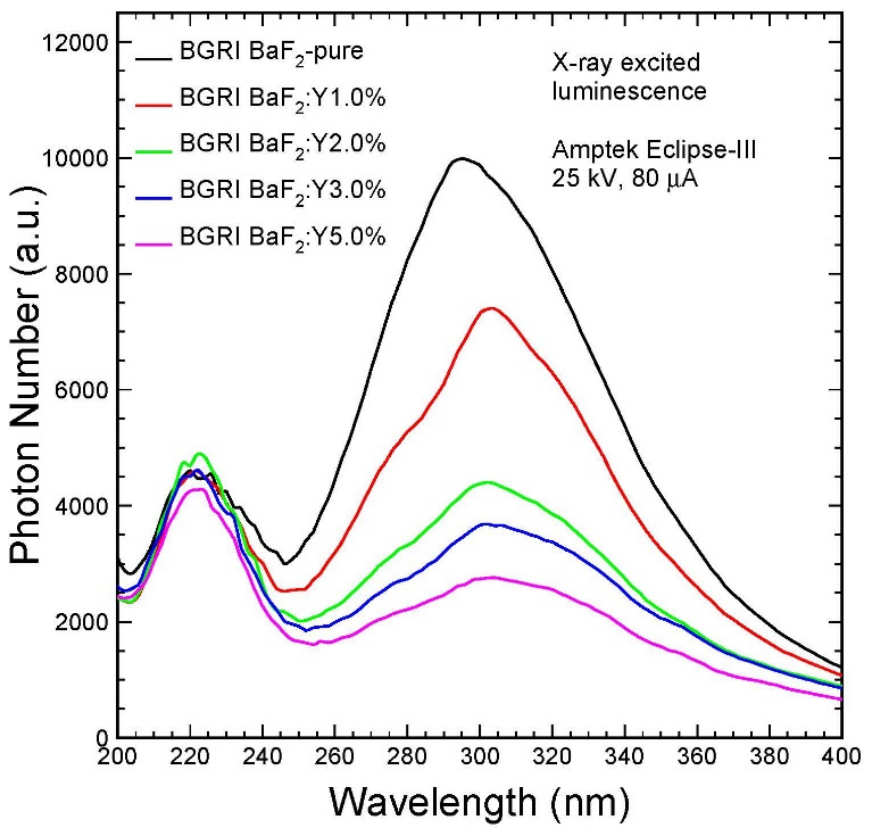

Fig. $1 \mathrm{X}$-ray excited emission spectra measured for $\mathrm{BaF}_{2}$ crystal samples with different yttrium doping level [31].

\section{EXPERIMENTAL SETUP AND PHOTODETECTORS}

Fig. 2 shows a test bench used to measure QE and PDE as a function of wavelength. An UV extended $150 \mathrm{~W}$ Xenon lamp was used as the light source. The fluctuation of the light source intensity was monitored by a photodiode mounted on the lamp

Liyuan Zhang, Chen Hu, James Oyang, Bertrand Echenard, David Hitlin, Xuebin Qiao, Jason Trevor and Ren-Yuan Zhu are with the HEP, California Institute of Technology, Pasadena, CA 91125, USA (e-mail: zhu@caltech.edu). 
house and corrected for through a lock-in amplifier (Stanford Research Systems SR810). A narrow wavelength band was selected by a monochromator, chopped by an optical chopper, and coupled to a $1 \mathrm{~mm}$ quartz fiber with $\mathrm{NA}=0.22$. The light pulses from the fiber were centered to a $\Phi 5 \mathrm{~mm}$ area of the photodetector to be measured. The response of the photodetector was scanned from 200 to $800 \mathrm{~nm}$ and was readout through a low noise pre-amplifier (Newport 70710) and the lock-in amplifier.

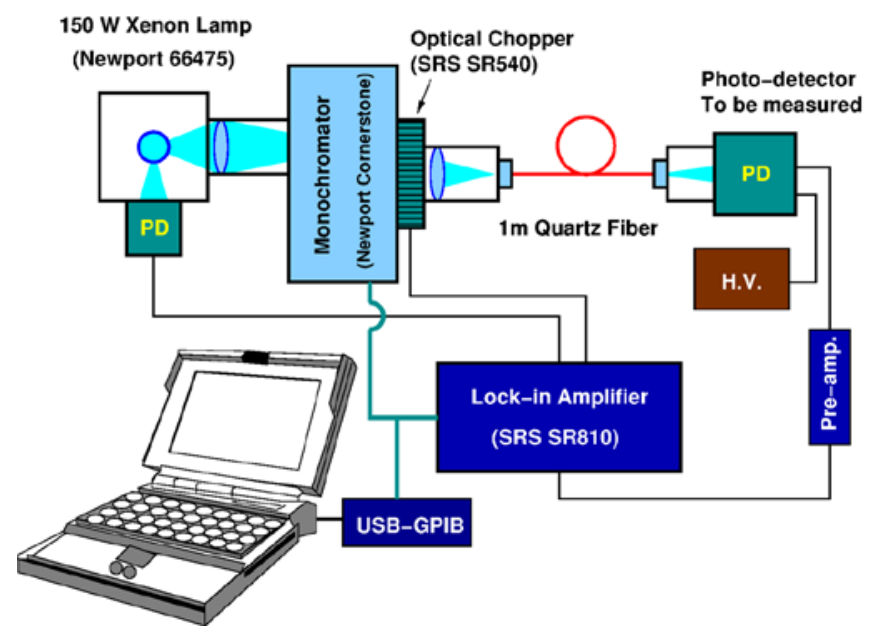

Fig. 2 A setup used to measure QE and PDE as a function of wavelength.

For photodetectors with a unit gain, their $Q E(\lambda)$ is obtained by normalizing its response $R(\lambda)$ to the response of a NIST traceable reference Si-PD (Newport 71650) $R_{P D}(\lambda)$ scanned under the same settings, as shown in Eq. 1:

$$
Q E(\lambda)=\frac{R(\lambda)}{R_{P D}(\lambda)} Q E_{P D}(\lambda)
$$

The QE of four Hamamatsu R2059 PMTs and a Photek solarblind photo-cathode diode was measured, where a home-made socket connected all dynodes to set their gain to one.

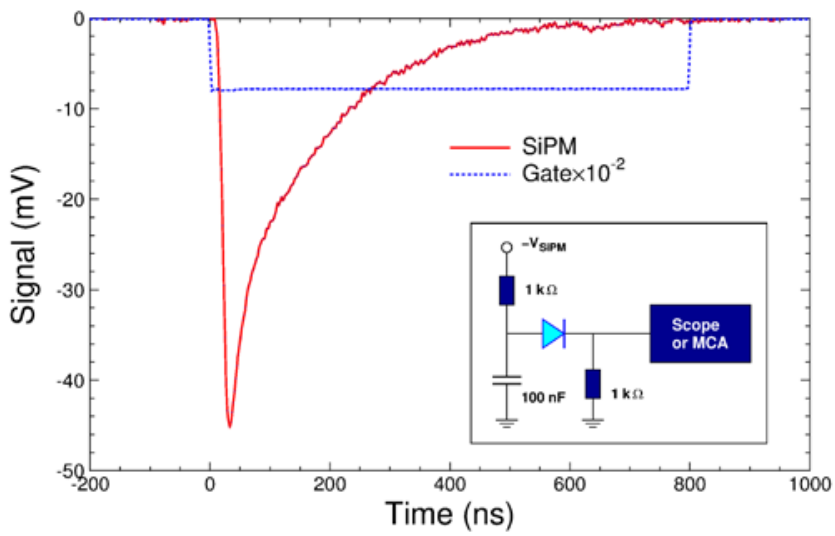

Fig. 3 SiPM output pulse (red) to $10 \mathrm{~ns}$ pulse from a $465 \mathrm{~nm}$ blue LED is shown in an 800 ns gate (blue dots) for pulse height spectra.

For photodetectors with gain, such as SiPM, the normalized response $R(\lambda)$ is a product of the PDE and the gain, so is a relative PDE. The calibration of SiPM PDE was carried out by using a $465 \mathrm{~nm}$ blue LED. Fig. 3 shows a scope trace of SiPM response (red) to LED pulses of 10 ns FWHM. The charge of the SiPM output pulses was integrated by a LeCroy 3001 qvt in 800 ns gate (blue dots) and digitized for a pulse height spectrum.

Figs. 4 and 5 show the pulse height spectra measured by two FBK R\&D SiPMs, sn612-A1 and sn615-A1, integrated with UV filters I and II respectively with breakdown voltages of 25.0 $\mathrm{V}$ and $31.5 \mathrm{~V}$. Also shown in the figures are the numerical values of the position and widths of photopeak and pedestal.

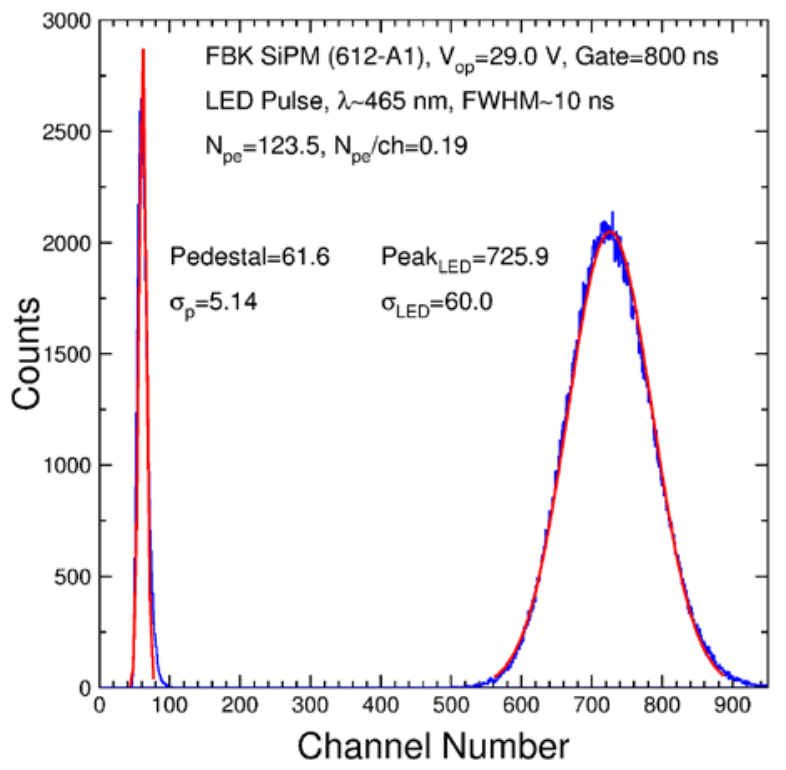

Fig. 4 The pulse height spectrum of a FBK SiPM (612-A1, $\left.\mathrm{V}_{\mathrm{br}}=25.0 \mathrm{~V}\right)$ with UV filter I measured by using $10 \mathrm{~ns}$ light pulse from a $465 \mathrm{~nm}$ LED.

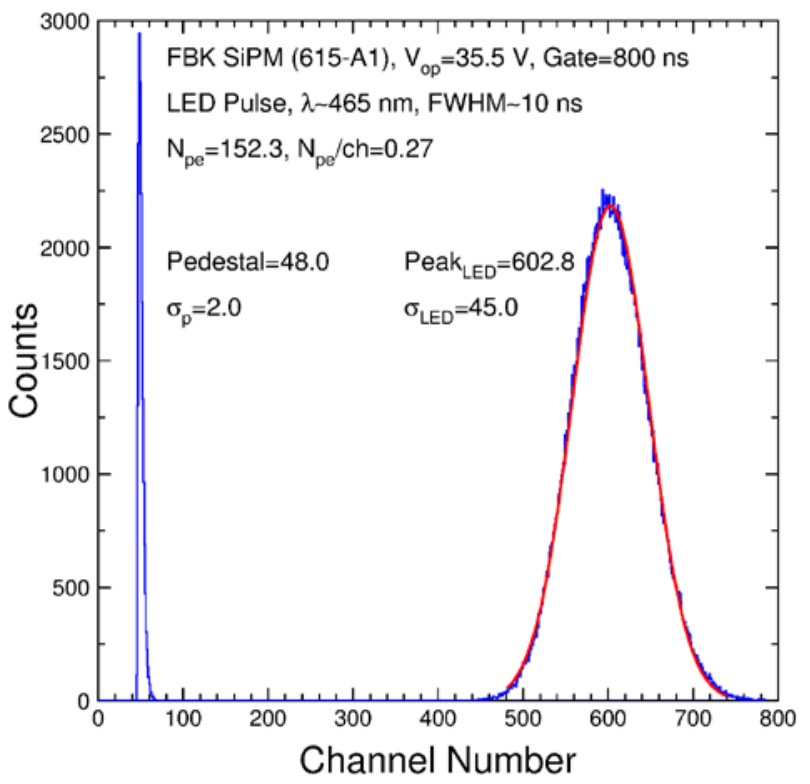

Fig. 5 The pulse height spectrum of FBK SiPM (615-A1, $V_{b r}=31.5$ V) with UV filter II measured by using $10 \mathrm{~ns}$ light pulse from a $465 \mathrm{~nm}$ LED.

The photoelectron (p.e.) number of the photopeak was calculated according to Eq. 2.

$$
N_{\text {p.e. }}=\frac{\left(P_{L E D}-P_{P}\right)^{2}}{\sigma_{L E D}{ }^{2}-\sigma_{P}{ }^{2}}
$$


where $P_{L E D}$ and $\sigma_{L E D}$ are the LED photo-peak and width, $P_{P}$ and $\sigma_{P}$ are the pedestal peak and width. The photoelectron number was determined as 123.5 and 152.3 for these two FBK SiPMs, respectively.

The input photon number from LED pulses was determined by measuring the DC current by a reference Si-PD with known QE according to Eq 3.

$$
N_{p h}=\frac{I_{P D} / S}{h v_{L E D} \times f_{L E D}}
$$

where $I_{P D}$ and $S$ are the photocurrent and sensitivity of the reference $\mathrm{Si}-\mathrm{PD}, h v_{\mathrm{LED}}$ and $f_{\mathrm{LED}}$ are the LED photon energy and the pulse rate.

The PDE value at $465 \mathrm{~nm}$ was determined as Eq. 4.

$$
P D E(465 \mathrm{~nm})=\frac{N_{p . e .}}{N_{p h}}
$$

The $\sigma_{L E D}$, obtained from the Gaussian fitting, however, is affected by the excess noise factor (ENF) of the photodetector. A cross-check was carried out by measuring the response of a Hamamatsu R2059 PMT with a gain provided by a normal base socket. The resultant ENF was found to be about 1.1, which is consistent with Hamamatsu technical note. The ENF values for SiPMs were also measured. Fig. 6 shows that individual photon electron peaks can be clearly resolved in SiPM response to attenuated LED pulses by using an amplifier (Ortec VT120) and digitizer (CAEN V1720). The ENF values were determined as the ratio between the photoelectron numbers from this single p.e. calibration to that from Eq. 2.

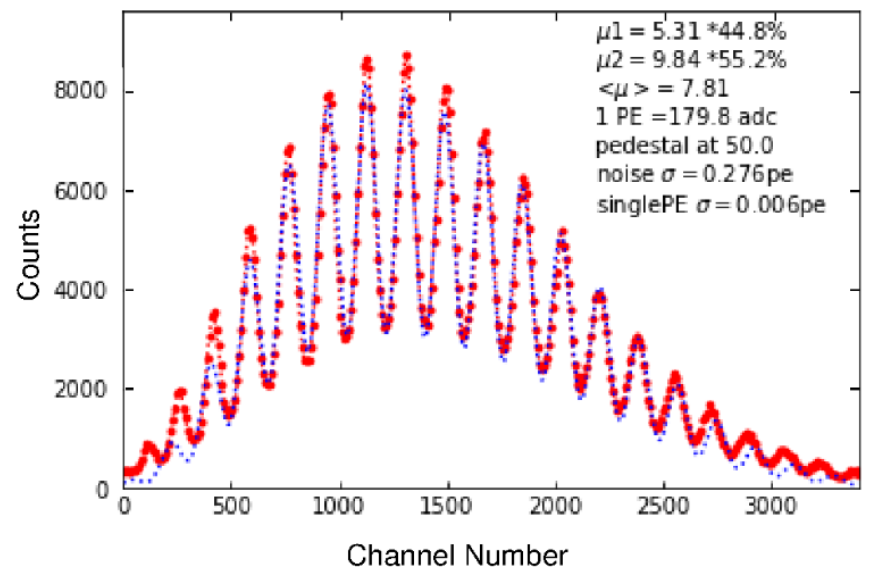

Fig. 6 Individual photo electron peaks observed by a FBK SiPM its responding to attenuated LED pulses.

After the ENF correction shown in Eq. 5, the PDE values at $465 \mathrm{~nm}$ are $6.3 \%$ and $35.1 \%$ for two FBK SiPMs with Type I and II filters, respectively.

$$
P D E(465 \mathrm{~nm})=\frac{N_{p . e .}}{N_{p h}} \times E N F
$$

The PDE of four Hamamatsu MPPCs (or SiPMs) and several FBK R\&D SiPMs with integrated UV filter was obtained by using the relative PDE $R(\lambda)$ from 200 to $600 \mathrm{~nm}$ and the 465 nm calibration.

\section{RESULTS AND DISCUSSION}

Fig. 7 shows consistent QE spectra between 200 and $700 \mathrm{~nm}$ for four Hamamatsu R2059 PMTs.

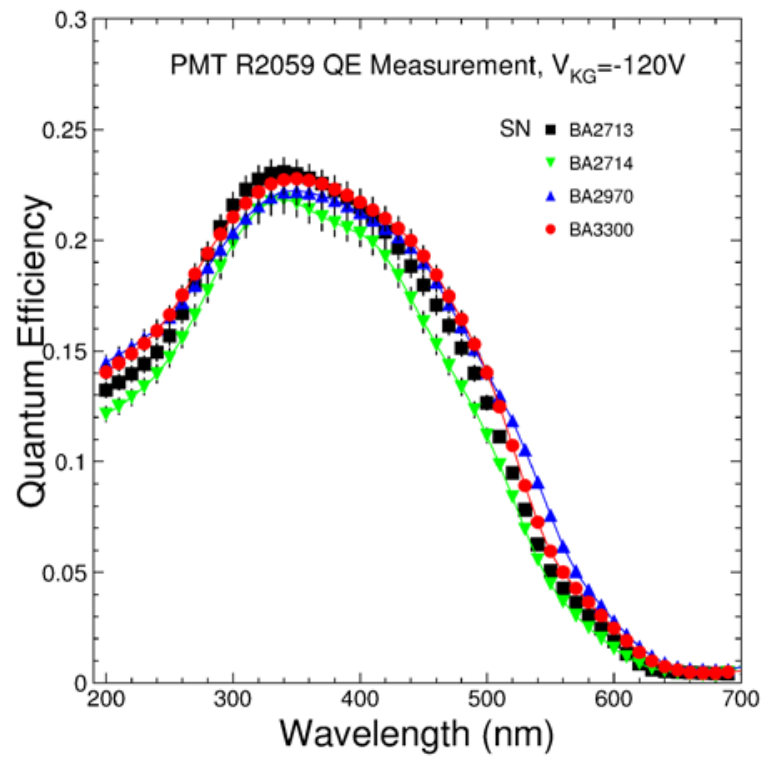

Fig. 7 The measured QE (200-700 nm) of four Hamamatsu R2059 PMTs.

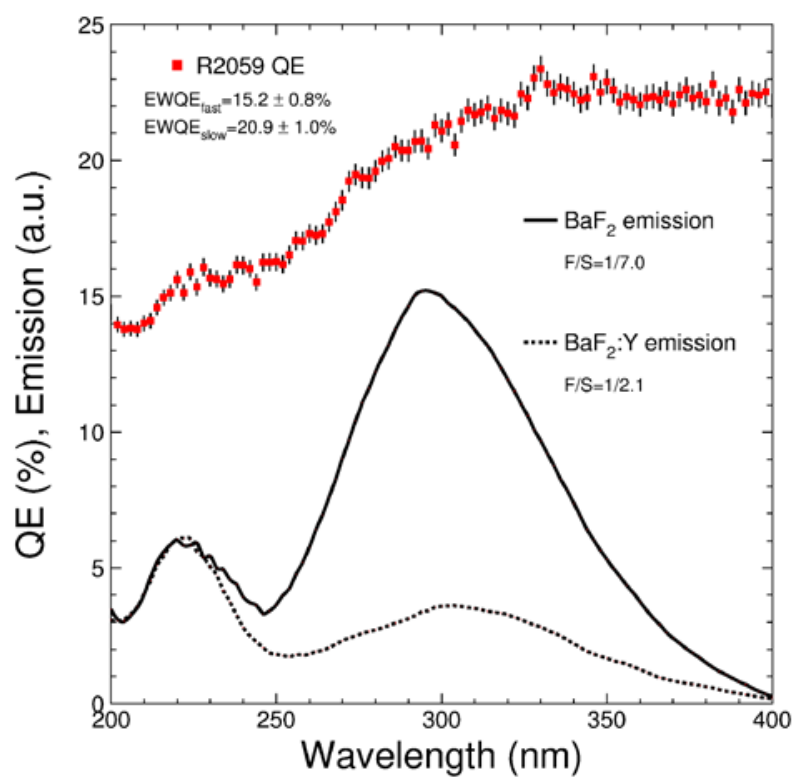

Fig. 8 The measured QE (200-400 nm) of a Hamamatsu R2059 PMT and the X-ray excited emission spectra of $\mathrm{BaF}_{2}$ and $\mathrm{BaF}_{2}: \mathrm{Y}$.

Fig. 8 shows the measured QE spectrum (red dots with error bars) between 200 and $400 \mathrm{~nm}$ for a R2059 PMT and the X-ray excited emission spectra of $\mathrm{BaF}_{2}$ (black solid lines) and $\mathrm{BaF}_{2}: \mathrm{Y}$ with suppressed slow emission (black dashed lines). Also shown in the figure are the numerical values of the emission weighted QE (EWQE) defined as: 


$$
E W Q E_{\text {fast or slow }}=\frac{\int_{\lambda_{1}}^{\lambda_{2}} E m(\lambda) Q E(\lambda) d \lambda}{\int_{\lambda_{1}}^{\lambda_{2}} E m(\lambda) d \lambda}
$$

where $\operatorname{Em}(\lambda)$ is the $\mathrm{x}$-ray excited emission spectrum of $\mathrm{BaF}_{2}$ or $\mathrm{BaF}_{2}: \mathrm{Y}$, and $\lambda_{1}=200$ and $250 \mathrm{~nm}$ and $\lambda_{2}=250$ and $400 \mathrm{~nm}$ respectively for the fast and slow light. The $\mathrm{EWQE}_{\text {fast }}$ and EWQE $_{\text {slow }}$ values are $15.2 \%$ and $20.9 \%$ respectively for the fast and slow light. Since the emission spectra of $\mathrm{BaF}_{2}$ and $\mathrm{BaF}_{2}: \mathrm{Y}$ have a similar line shape (with different intensity for the slow light) the corresponding emission weighted $\mathrm{QE}$ values are the same for both $\mathrm{BaF}_{2}$ and $\mathrm{BaF}_{2}: \mathrm{Y}$. Also shown in the figure are the ratio between the fast and the slow scintillation light $(\mathrm{F} / \mathrm{S})$ for $\mathrm{BaF}_{2}$ and $\mathrm{BaF}_{2}: \mathrm{Y}$, which are defined as:

$$
F / S=\frac{\int_{200 n m}^{250 n m} E m(\lambda) Q E(\lambda) d \lambda}{\int_{250 n m}^{400 n m} E m(\lambda) Q E(\lambda) d \lambda}
$$

The $\mathrm{EWQE}_{\text {fast }}$ and the $\mathrm{F} / \mathrm{S}$ ratio are considered as the figures of merit representing photodetector's ability for the fast detection and the slow suppression, respectively. While the EWQE $E_{\text {fast }}$ values are 0.15 for both $\mathrm{BaF}_{2}$ and $\mathrm{BaF}_{2}: \mathrm{Y}$, their $\mathrm{F} / \mathrm{S}$ ratio values are $1 / 7$ and $1 / 2.1$ respectively for the Hamamatsu R2059, indicating a factor of 3.5 improvement in the slow suppression provided by $\mathrm{BaF}_{2}: \mathrm{Y}$.

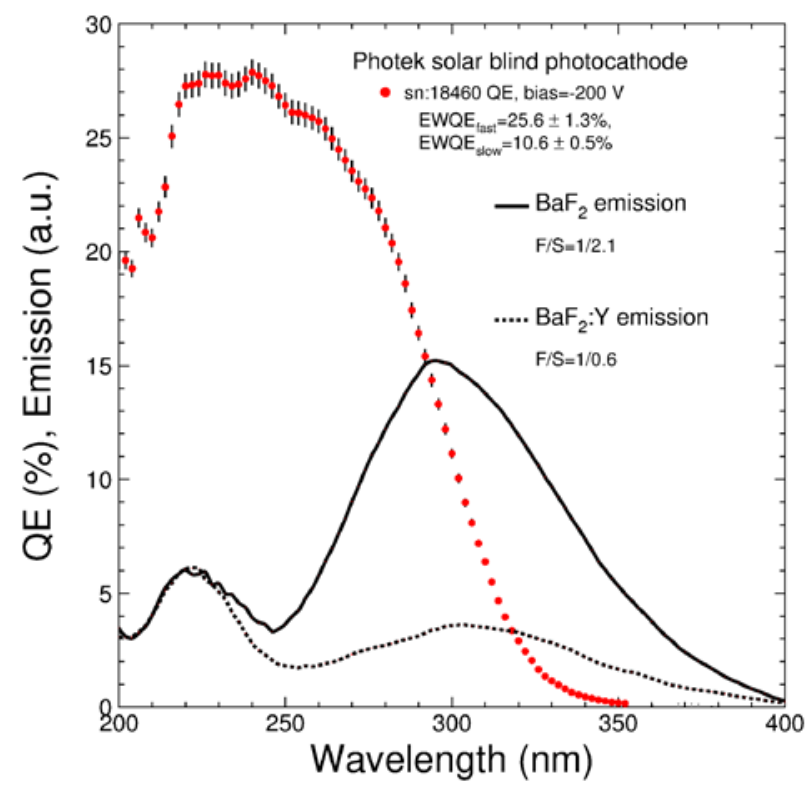

Fig. 9 The measured QE (200-400 nm) of Photek solar blind photocathode and the $\mathrm{X}$-ray excited emission spectra of $\mathrm{BaF}_{2}$ and $\mathrm{BaF}_{2}: \mathrm{Y}$.

Fig. 9 shows the measured QE between 200 and $400 \mathrm{~nm}$ for the Photek solar blind photocathode. The EWQE for fast and slow components are $25.6 \%$ and $10.6 \%$ respectively, showing a high detection efficiency and a clear selective readout for the fast component. While the $\mathrm{EWQE}_{\text {fast }}$ values are 0.26 for both $\mathrm{BaF}_{2}$ and $\mathrm{BaF}_{2}: \mathrm{Y}$, the $\mathrm{F} / \mathrm{S}$ values are $1 / 0.6$ and $1 / 2.1$, indicating once again a factor of 3.5 better slow suppression for $\mathrm{BaF}_{2}: \mathrm{Y}$. Compared to the bi-alkali cathode of Hamamatsu 2059 shown in Fig. 10, the Photek solar-blind cathode shows a factor of 1.5 better efficiency for fast detection and another factor of 3.5 better for slow suppression.

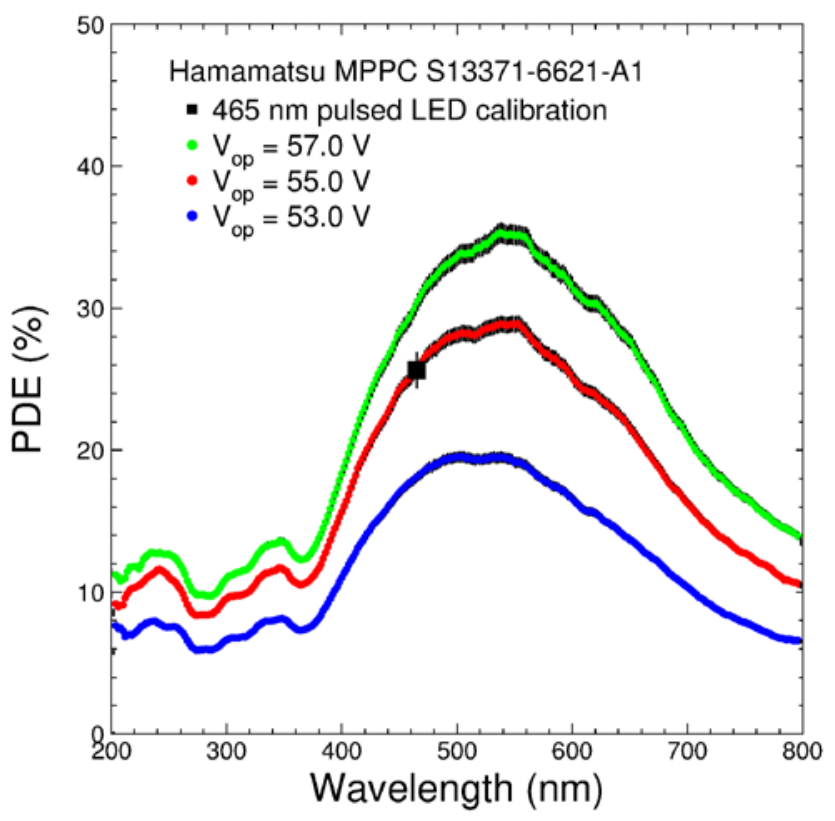

Fig. 10 The measured PDE (200-800 nm) of the Hamamatsu VUV3 MPPC $\left(\mathrm{S} 13371-6621-\mathrm{A} 1, \mathrm{~V}_{\mathrm{br}}=51.0 \mathrm{~V}\right.$ ) at operation voltage of 53, 55 and $57 \mathrm{~V}$.

Fig. 10 shows PDE for the Hamamatsu VUV3 MPPC S13371-6621-A1 ( $\left.\mathrm{V}_{\mathrm{br}}=51.0 \mathrm{~V}\right)$ between 200-800 $\mathrm{nm}$ with three operation voltage of 53 (blue), 55 (red) and 57 (green) V. Also shown in the figure is the $465 \mathrm{~nm}$ blue LED calibration at the nominal operating voltage $55 \mathrm{~V}$.

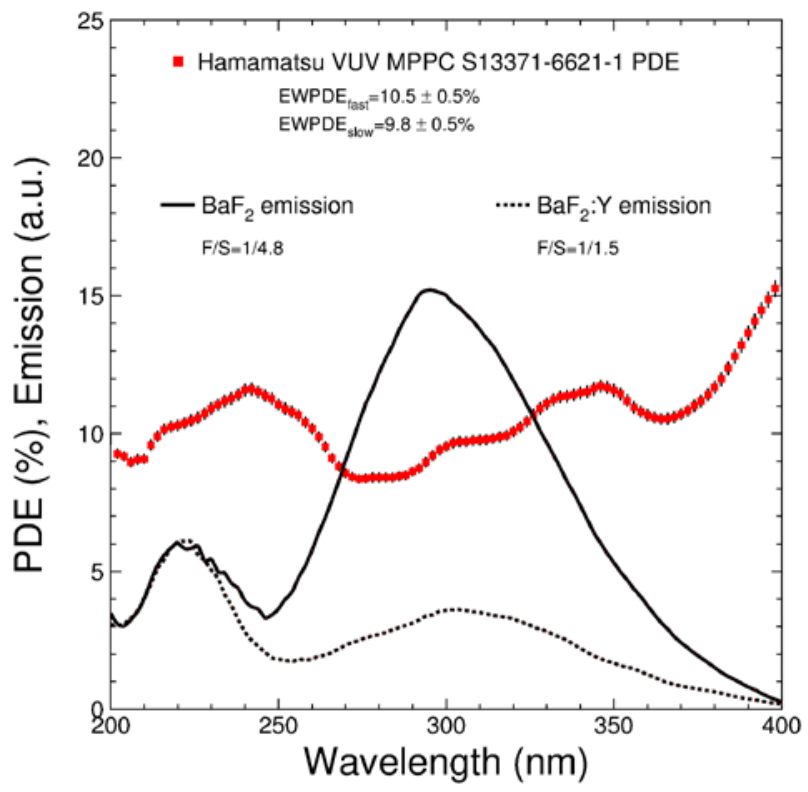

Fig. 11 The measured PDE (200-400 nm) of the Hamamatsu VUV3 MPPC and the X-ray excited emission spectra of $\mathrm{BaF}_{2}$ and $\mathrm{BaF}_{2}: \mathrm{Y}$.

Fig. 11 shows PDE between 200 and $400 \mathrm{~nm}$ for the Hamamatsu VUV3 MPPC S13371-6621-A1 at the nominal operating voltage $55.0 \mathrm{~V}$. Its low $\mathrm{EWQE}_{\text {fast }}$ values of 0.10 indicates a low detection efficiency for the fast component. Its $\mathrm{F} / \mathrm{S}$ values are $1 / 4.8$ and $1 / 1.5$ respectively for $\mathrm{BaF}_{2}$ and $\mathrm{BaF}_{2}: \mathrm{Y}$ 
crystals respectively, indicating a factor of 3.2 better slow suppression for $\mathrm{BaF}_{2}: \mathrm{Y}$. Compared to $\mathrm{PMTs}$, its $\mathrm{F} / \mathrm{S}$ ratio is better than bi-alkali cathode (R2059), but worse than Photek solar-blind cathode.

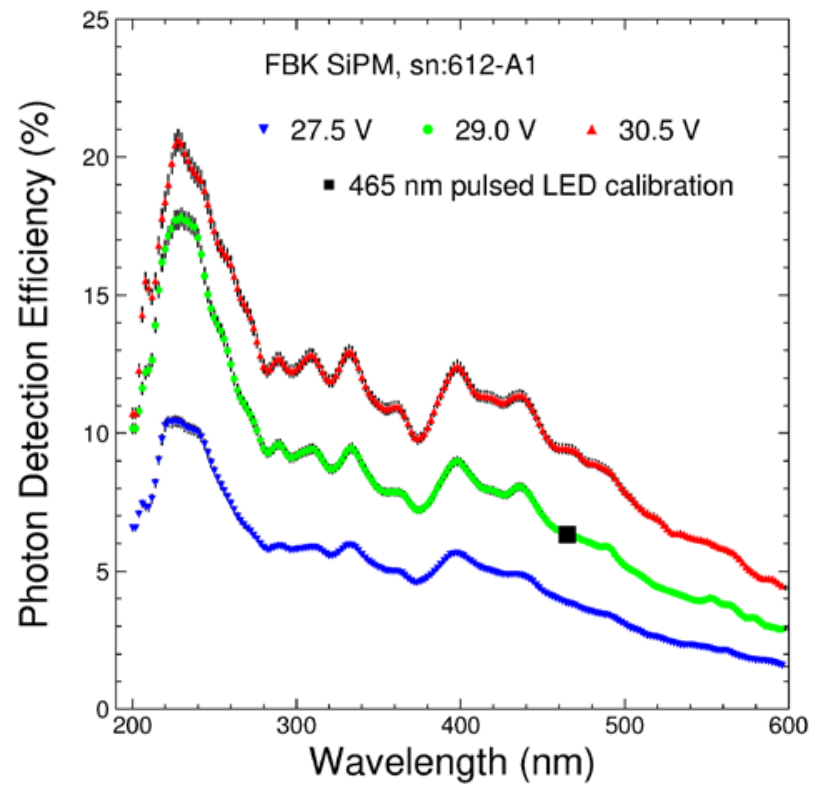

Fig. 12 The measured PDE (200-600 nm) of FBK SiPM sn:612-A1 with the UV filter I at operation voltage of 27.5, 29.0 and $30.5 \mathrm{~V}, \mathrm{~V}_{\mathrm{br}}=25.0 \mathrm{~V}$.

Fig. 12 shows measured PDE spectra between 200 and 600 $\mathrm{nm}$ for the FBK SiPM integrated with the type I UV bandpass filter (sn:612-A1, $\mathrm{V}_{\mathrm{br}}=25.0 \mathrm{~V}$ ) at operation voltage of 27.5, 29.0 and $30.5 \mathrm{~V}$. Also shown in the figure is the $465 \mathrm{~nm}$ blue LED calibration done at the nominal operating voltage $29 \mathrm{~V}$.

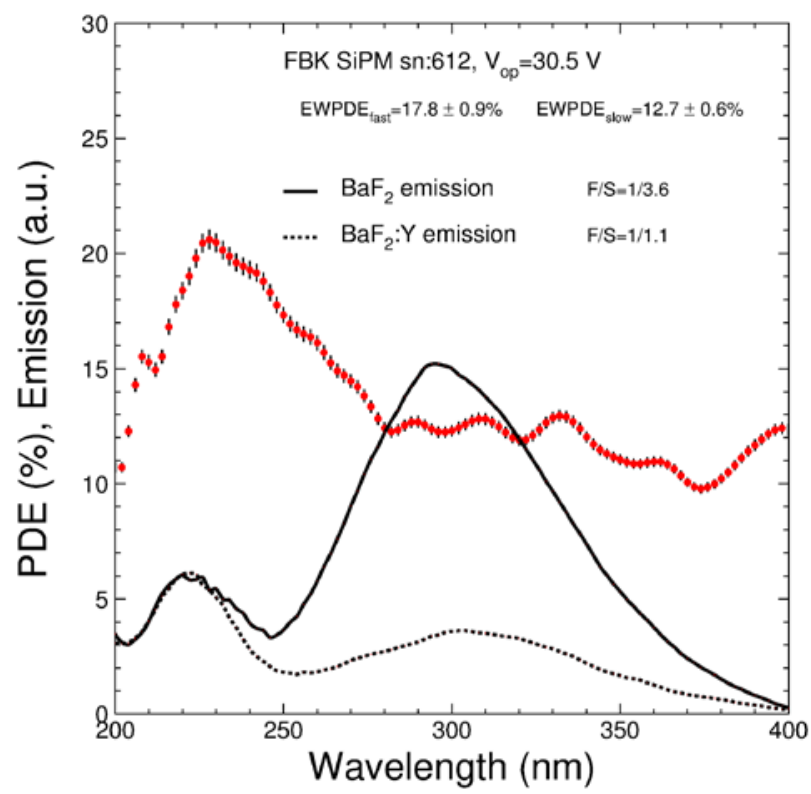

Fig. 13 The measured PDE (200-400 nm) of the FBK SiPM 612-A1 at 30.5V and the $\mathrm{X}$-ray excited emission spectra of $\mathrm{BaF}_{2}$ and $\mathrm{BaF}_{2}: \mathrm{Y}$.

Fig. 13 shows its PDE (200-400 nm) at 30.5V and the X-ray excited emission spectra of $\mathrm{BaF}_{2}$ and $\mathrm{BaF}_{2}: \mathrm{Y}$. The emission weighted photon detection efficiencies (EWPDE) are 17.8\% and $12.7 \%$ for the fast and slow component. The figures of merit are 0.18 for the fast component detection and 1/3.6 and 1/1.1 for the $\mathrm{F} / \mathrm{S}$ ratio for $\mathrm{BaF}_{2}$ and $\mathrm{BaF}_{2}: \mathrm{Y}$ respectively, indicating a promising solution for slow suppression. $\mathrm{R} \& \mathrm{D}$ will be continued along this direction.

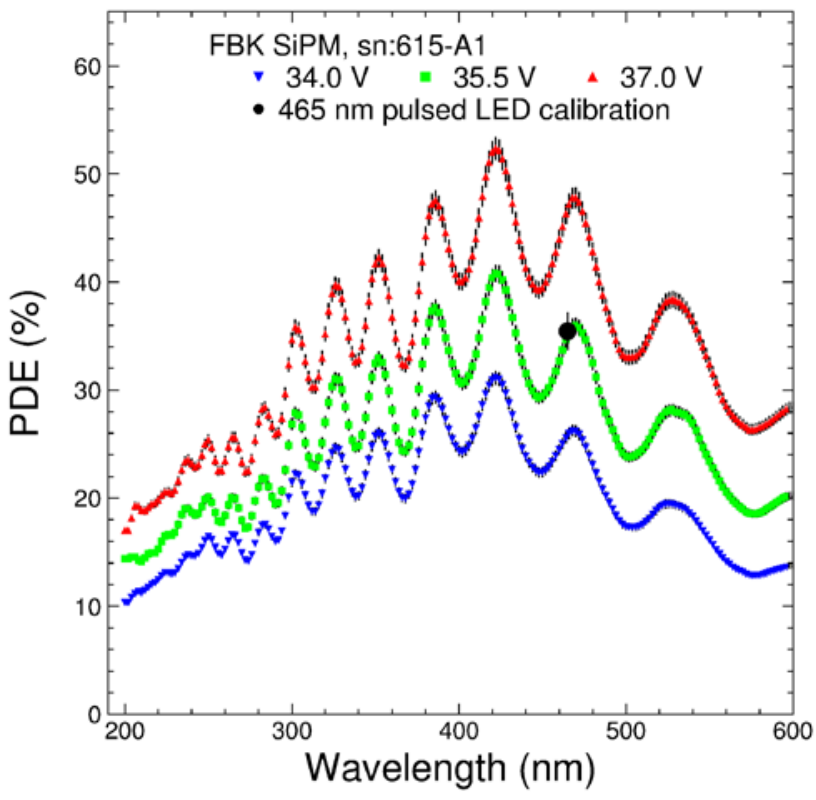

Fig. 14 The measured PDE (200-600 nm) of FBK SiPM sn:615-A1 with the UV filter II at operation voltage of $34.0,35.5$ and $37.0 \mathrm{~V}, \mathrm{~V}_{\mathrm{br}}=31.5 \mathrm{~V}$.

Fig. 14 shows the measured PDE spectra between 200 and $600 \mathrm{~nm}$ for the FBK SiPM integrated with the type II UV filter (sn:615-A1, $\mathrm{V}_{\mathrm{br}}=31.5 \mathrm{~V}$ ) at operation voltage of 34.0, 35.5 and $37.0 \mathrm{~V}$. The observed oscillation is consistent with the filter design. Also shown in the figure is the $465 \mathrm{~nm}$ blue LED calibration at the nominal operation voltage $35.5 \mathrm{~V}$.

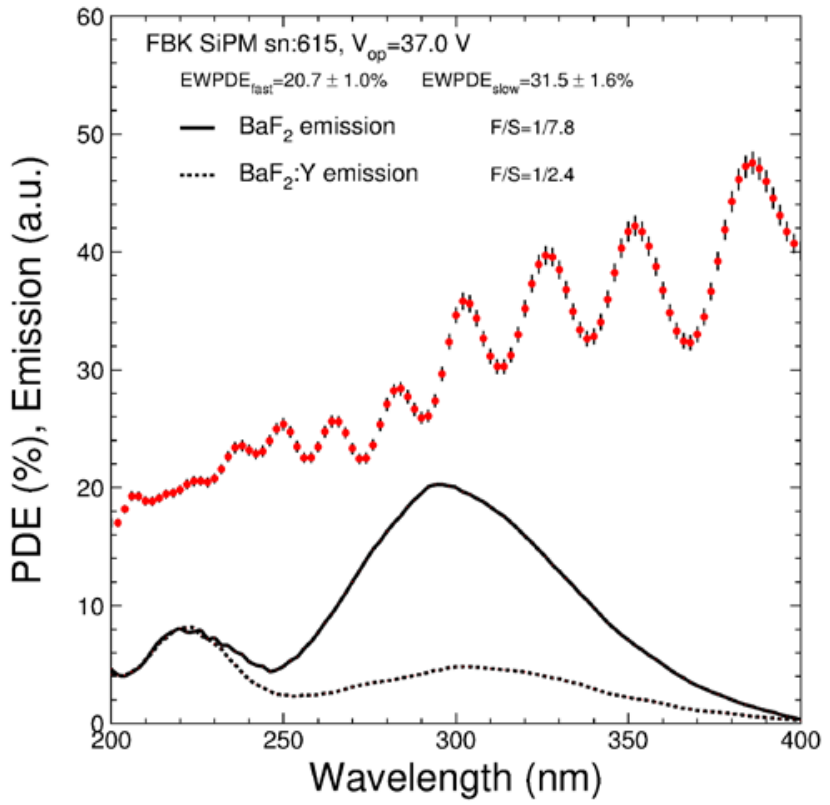

Fig. 15 The measured PDE (200-400 nm) of the FBK SiPM 615-A1 at 37.0 $\mathrm{V}$ and the $\mathrm{X}$-ray excited emission spectra of $\mathrm{BaF}_{2}$ and $\mathrm{BaF}_{2}: \mathrm{Y}$. 
Fig. 15 shows its PDE (200-400 nm) spectrum at 37V and the X-ray excited emission spectra of $\mathrm{BaF}_{2}$ and $\mathrm{BaF}_{2}: \mathrm{Y}$. The spectral response shows no additional slow suppression with this type of UV filter. R\&D will not be continued along this direction.

Table I summarized the numerical values for the photodetectors investigated. The first two columns are EWQE/ EWPDE for the fast and the slow component. The third and fourth ones are the fast/slow ratio for $\mathrm{BaF}_{2}$ and $\mathrm{BaF}_{2}: \mathrm{Y}$ crystals, where the slow suppression effect of $\mathrm{BaF}_{2}: \mathrm{Y}$ is shown. While the EWQE/EWPDE is directly related to the light output from a photodetector, the F/S ratio represents its ability for slow suppression. A combination of $\mathrm{BaF}_{2}: \mathrm{Y}$ crystals and selective readout provided by solar-blind photodetectors from either Photek photocathode or FBK SiPM integrated with the type-I UV bandpass filter shows a promising solution.

\begin{tabular}{ccccc}
\multicolumn{5}{c}{ TABLE I. SUMMARY OF THE FIGURE OF MERIT FOR PHOTODETECTORS } \\
\hline Photodetectors & $\begin{array}{c}\mathrm{EWQE}_{\text {fast }} \\
(\%)\end{array}$ & $\begin{array}{c}\text { EWQE } \\
\text { slow }\end{array}$ & $\mathrm{BaF}_{2}$ & $\mathrm{BaF}_{2}: \mathrm{Y}$ \\
Hamamatsu & 15.2 & 20.9 & $1 / 7.0$ & $1 / 2.1$ \\
R2059 PMT & & & & \\
$\begin{array}{c}\text { Hamamatsu } \\
\text { MPPC S13371 }\end{array}$ & 10.5 & 9.8 & $1 / 4.8$ & $1 / 1.5$ \\
$\begin{array}{c}\text { Photek PMT } \\
\text { Solar blind }\end{array}$ & $\mathbf{2 5 . 6}$ & $\mathbf{1 0 . 6}$ & $\mathbf{1 / 2 . 1}$ & $\mathbf{1 / 0 . 6}$ \\
$\begin{array}{c}\text { FBK SiPM } \\
\text { w/UV filter-I }\end{array}$ & $\mathbf{1 7 . 8}$ & $\mathbf{1 2 . 7}$ & $\mathbf{1 / 3 . 6}$ & $\mathbf{1 / 1 . 1}$ \\
$\begin{array}{c}\text { FBK SiPM } \\
\text { W/UV filter-II }\end{array}$ & 20.7 & 31.5 & $1 / 7.8$ & $1 / 2.4$ \\
\hline
\end{tabular}

\section{SUMMARY}

While yttrium doping in $\mathrm{BaF}_{2}$ crystals increases the $\mathrm{F} / \mathrm{S}$ ratio by a factor of three, solar-blind photodetectors would further reduce pileup noise for a $\mathrm{BaF}_{2}$ crystal based ultrafast calorimeter for future HEP experiments, such as Mu2e-II. Progresses have been made in both solar-blind photocathode and SiPMs with integrated UV band-pass filter. R\&D is ongoing to further optimize the EWQE/EWPDE $\mathrm{E}_{\text {fast }}$ and the $\mathrm{F} / \mathrm{S}$ ratio for $\mathrm{BaF}_{2}$ crystal readout with photodetectors.

Since a fraction of $\mathrm{BaF}_{2}$ emission is below $200 \mathrm{~nm}$ we plan to extend the coverage of our QE/PDE test bench to the VUV range down to $175 \mathrm{~nm}$ with either vacuum or $\mathrm{N}_{2}$ purging. Novel VUV photodetectors, such as the Hamamatsu SiPM investigated in this manuscript and other semiconductor-based VUV photodetectors $[37,38]$ will also be investigated.

\section{ACKNOWLEDGEMENTS}

The authors are grateful to Dr. P. Hink for providing the solar-blind photocathode investigated in this work.

\section{REFERENCES}

[1] N. N. Ershov, N. G. Zakharov, P. A. Rodnyi, "Spectral-kinetic study of the intrinsic-luminescence characteristics of a fluorite-type crystal," Opt. Spectrosc., vol. 53, no. 1, pp. 51-54, Jul. 1982.

[2] M. Laval, M. Moszynski, R. Alleman, E. Cormoreche, P. Guinet, R. Odru and J. Vacher, "Barium fluoride-inorganic scintillator for subnanosecond timing,” Nucl. Instrum. Methods Phys. Res., vol. 206, no. 1-2, pp. 169-176, Feb. 1983.

[3] Y. M. Aleksandrov, V. N. Makhov, P. A. Rodnyj, T. I. Syrejshchikova, and M. N. Yakimenko, "Intrinsic luminescence of $\mathrm{BaF}_{2}$ at pulsed synchrotron radiation excitation,” Sov. Phys. Solid State, vol. 26, no. 9, pp. 2865-2867, 1984.

[4] Ya. A. Valbis, Z. A. Rachko, and Ya. L. Yansons, "Short-wave UV luminescence of $\mathrm{BaF}_{2}$ crystals caused by crossover transitions," Soviet $J$. Exp. Theor. Phys. Lett., vol. 42, no. 4, pp. 140-142, Aug. 1985.

[5] P. Dorenbos, R. Visser, J. Andriessen, C. W. E. van Eijk, J. Valbis, and N. M. Khaidukov, "Scintillation properties of possible cross luminescence materials,” Nucl. Tracks Radiat. Meas., vol. 21, no. 1, pp. 101-103, Jan. 1993.

[6] V. N. Makhov, "Vacuum ultraviolet luminescence of wide band-gap solids studied using time-resolved spectroscopy with synchrotron radiation,” Phys. Scripta, vol. 89, no. 4, Feb. 2014, Art. no. 044010.

[7] J. Andriessen, P. Dorenbos, and C. W. E Van Eijk, "Electronic structure and transition probabilities in pure and $\mathrm{Ce}^{3+}$ doped $\mathrm{BaF}_{2}$, an explorative study,” Mol. Phys., vol. 74, no. 3, pp. 535-546, June 1991.

[8] S. E. Derenzo, M. J. Weber, W. W. Moses, and C. Dujardin, "Measurements of the Intrinsic Rise Times of Common Inorganic Scintillators," IEEE Trans. Nucl. Sci., vol. 47, no. 3, pp. 860-864, June 2000.

[9] C. Hu, L. Zhang, R.-Y. Zhu, A. Chen, Z. Wang, L. Ying, and Z. Yu, "Ultrafast Inorganic Scintillators for Gigahertz Hard X-Ray Imaging," IEEE Trans. Nucl. Sci., vol. 65, no. 8, pp. 2097-2104, Aug. 2018.

[10] S. Gundacker, R. Martinez Turtos, N. Kratochwil, R. H. Pots, M. Paganoni, P. Lecoq, and E. Auffray, "Experimental time resolution limits of modern SiPMs and TOF-PET detectors exploring different scintillators and Cherenkov emission,” Phys. Med. Biol., vol. 65, no. 2, pp. 025001, Jan. 2020.

[11] R. H. Pots, E. Auffray, S. Gundacker, "Exploiting Cross-Luminescence in $\mathrm{BaF}_{2}$ for Ultrafast Timing Applications Using Deep-Ultraviolet Sensitive HPK Silicon Photomultipliers,” Front. Phys., vol. 8, pp. 482, Oct. 2020.

[12] R. Novotny, "Performance of the $\mathrm{BaF}_{2}$-calorimeter TAPS ${ }^{1}$," Nucl. Phys. B (Proc. Suppl.), vol. 61, no. 3, pp. 137-142, Feb. 1998.

[13] R.W. Garnett, M.S. Gulley, "Matter-radiation interactions in extremes", in Proc. Linear Accelerator Conf. (LINA), 2010, 485-487.

[14] G. Pezzullo et al., "Progress status for the Mu2e calorimeter system,” J. Phys., Conf. Ser., vol. 587, no. 1, 2015, Art. no. 012047.

[15] N. Atanov et al., "Design and status of the Mu2e crystal calorimeter," IEEE Trans. Nucl. Sci., vol. 65, no. 8, pp. 2073-2080, Aug. 2018.

[16] F. Yang, L. Zhang, and R.-Y. Zhu, "Gamma-ray induced radiation damage up to $340 \mathrm{Mrad}$ in various scintillation crystals," IEEE Trans. Nucl. Sci., vol. 63, no. 2, pp. 612-619, Apr. 2016.

[17] C. Hu, F. Yang, L. Zhang, R.-Y. Zhu, J. Kapustinsky, R. Nelson, and Zhehui Wang, "Proton-induced radiation damage in $\mathrm{BaF}_{2}, \mathrm{LYSO}$, and PWO crystal scintillators," IEEE Trans. Nucl. Sci., vol. 65, no. 4, pp. 1018-1024, Apr. 2018.

[18] C. Hu, F. Yang, L. Zhang, R.-Y. Zhu, J. Kapustinsky, M. Mocko, R. Nelson, and Zhehui Wang, "Neutron-induced radiation damage in $\mathrm{BaF}_{2}$, LYSO and PWO crystals," IEEE Trans. Nucl. Sci., vol. 67, no. 6, pp. 1086-1092, Jun. 2020.

[19] F. Abusalma, D. Ambrose, A. Artikov, et al., "Expression of Interest for Evolution of the Muto 2e Experiment," arXiv:1802.02599v1.

[20] P. Schotanus, P. Dorenbos, C. W. E. van Eijk, and H. J. Lamfers, "Suppression of the slow scintillation light output of $\mathrm{BaF}_{2}$ crystals by $\mathrm{La}^{3+}$ doping,” Nucl. Instrum. Methods Phys. Res. A, Accel. Spectrom. Detect. Assoc. Equip., vol. 281, pp. 162-166, Aug. 1989.

[21] C. L. Woody, P. W. Levy, and J. A. Kierstead, "Slow component suppression and radiation damage in doped $\mathrm{BaF}_{2}$ crystals," IEEE Trans. Nucl. Sci., vol. 36, no. 1, pp. 536-542, Feb. 1989.

[22] P. A. Rodnyi, M. A. Terekhin, and E. N. Mel'chakov, "Radiative corevalence transitions in barium-based fluorides," J. Lumin., vol. 47, no. 6, pp. 281-284, Feb. 1991. 
[23] B. P. Sobolev, E. A. Krivandina, S. E. Derenzo, and W. W. Moses, "Suppression of BaF2 slow component of $\mathrm{X}$-ray luminescence in nonstoichiometric $\mathrm{Ba}_{0.9} \mathrm{R}_{0.1} \mathrm{~F}_{2.1}$ crystals ( $\mathrm{R}=$ rare earth element)," Proc. Mater. Res. Soc., Scintillator Phosphor Mater., vol. 348, pp. 227-283, 1994.

[24] A. I. Nepomnyashchikh, E.A. Radzhabov, A.V. Egranov, V.F. Ivashechkin, A.S. Istomin, T. Kurobori, "Defect formation in $\mathrm{BAF}_{2}$ crystals doped with cadmium,” Nucl. Instrum. Methods Phys. Res. A, Accel. Spectrom. Detect. Assoc. Equip., vol. 537, no. 1-2, pp. 27-30, 2005.

[25] E. Radzhabov, A. Istomin, A. Nepomnyashikh, A. Egranov, and V. Ivashechkin, "Exciton interaction with impurity in barium fluoride crystals," Nucl. Instrum. Methods Phys. Res. A, Accel. Spectrom. Detect. Assoc. Equip., vol. 537, pp. 71-75, Jan. 2005.

[26] A. S. Myasnikova, E. A. Radzhabov, and A. V. Egranov, "Extrinsic luminescence of $\mathrm{BaF}_{2}: \mathrm{R}^{3+}$ crystals $\left(\mathrm{R}^{3+}=\mathrm{La}^{3+}, \mathrm{Y}^{3+}, \mathrm{Yb}^{3+}\right)$," Phys. Solid State, vol. 50, pp. 1644-1647, Sep. 2008.

[27] S. Diehl, R. W. Novotny, B. Wohlfahrt and R. Beck, "Readout concepts for the suppression of the slow component of $\mathrm{BaF}_{2}$ for the upgrade of the TAPS spectrometer at ELSA,” J. Phys. Conf. Ser., vol. 587, pp. 012044, Feb. 2015.

[28] F. Yang, J. Chen, L. Zhang, C. Hu, and R.-Y. Zhu, "La and La/Ce doped $\mathrm{BaF}_{2}$ crystals for future HEP experiments at the energy and intensity frontiers part I,” IEEE Trans. Nucl. Sci., vol. 66, no. 1, pp. 506-511, Jan. 2019.

[29] F. Yang, J. Chen, L. Zhang, C. Hu, and R.-Y. Zhu, "La and La/Ce doped $\mathrm{BaF}_{2}$ crystals for future HEP experiments at the energy and intensity frontiers part II,” IEEE Trans. Nucl. Sci., vol. 66, no. 1, pp. 512-518, Jan. 2019.

[30] J. Chen, F. Yang, L. Zhang, R.-. Zhu, Y. Du, S. Wang, S. Sun, and X. Li, "Slow scintillation suppression in yttrium doped $\mathrm{BaF}_{2}$ crystals," IEEE Trans. Nucl. Sci., vol. 65, no. 8, pp. 2147-2151, Aug. 2018.

[31] C. Hu, C. Xu, L. Zhang, Q. Zhang, and R.-Y. Zhu, "Development of Yttrium-Doped $\mathrm{BaF}_{2}$ Crystals for Future HEP Experiments," IEEE Trans. Nucl. Sci., vol. 66, no. 7, pp. 1854-1860, Jul. 2019.

[32] S. Gundacker, R. H. Pots, A. Nepomnyashchikh, E. Radzhabov, R. Shendrik, S. Omelkov, M. Kirm, F. Acerbi, M. Capasso, G. Paternoster, A. Mazzi, A. Gola, J. Chen, and E. Auffray, "Vacuum ultraviolet silicon photomultipliers applied to $\mathrm{BaF}_{2}$ cross-luminescence detection for highrate ultrafast timing applications,” Phys. Med. Biol., vol. 66, no. 11, pp. 114002, Jun. 2021.

[33] Z. Y. Wei, R. Y. Zhu, H, Newman, and Z. W. Yin, "Light yield and surface treatment of barium fluoride crystals," Nucl. Instrum. Methods Phys. Res. B, Beam Interact. Mater. At., vol. 61, pp. 61-66, Jul. 1991.

[34] R.-Y. Zhu, "On quality requirements to the barium fluoride crystals," Nucl. Instrum. Methods Phys. Res. A, Accel. Spectrom. Detect. Assoc. Equip., vol. 340, pp. 442-457, Mar. 1994.

[35] D. Hitlin, "Progress on a photosensor for the readout of the fast scintillation light component of $\mathrm{BaF}_{2}$," Talk presented in the 2021 CPAD workshop at Stony Brook, NY, March 2021. Accessible on the Web: https://indico.fnal.gov/event/46746/contributions/210202/attachments/14 1121/177609/Hitlin_CPAD_210318-.pdf.

[36] C. Hu, L. Zhang, and R.-Y. Zhu, "Development of Novel Inorganic Scintillators for Future High Energy Physics Experiments,” Talk presented in the 2021 CPAD workshop at Stony Brook, NY. On the Web: http://www.hep.caltech.edu/ zhu/talks/4Mu2eII_210303_BaF.pdf

[37] W. Zheng, L. Jia, and F. Huang, "Vacuum-Ultraviolet Photon Detections,” iScience, vol. 23, no. 6, pp. 101145, June 2020.

[38] L. Jia, W. Zheng, and F. Huang, "Vacuum-ultraviolet photodetectors," PhotoniX, vol. 1, no. 1, pp. 22, Nov. 2020. 